\title{
SOMATIC EMBRYOGENESIS AND PLANT REGENERATION IN CAROB (CERATONIA SILIQUA L.)
}

\author{
JORGE M. CANHOTO*, SANDRA C. RAMA, AND GIL S. CRUZ \\ Instituto do Ambiente e Vida, Departamento de Botânica, Faculdade de Ciências e Tecnologia, \\ Universidade de Coimbra, 3001-455 Coimbra, Portugal
}

(Received 21 July 2005; accepted 14 August 2006; editor N. A. Reichert)

\begin{abstract}
Summary
Somatic embryos of carob (Ceratonia siliqua L.) were induced from cotyledonary segments excised from immature seeds when cultured on Murashige and Skoog media supplemented with several combinations of 6-benzylaminopurine (BA) and indole-3-butyric acid (IBA). The best frequencies of induction (33.8\%) were obtained when $4.4 \mu M \mathrm{BA}$ and $0.5 \mu M$ IBA were used. Shoots were also sporadically formed in the same media. When IBA was replaced by other auxins in the induction media, only $\alpha$-naphthaleneacetic acid (NAA) and indole-3-acetic acid (IAA) could induce somatic embryogenesis, although at lower rates than IBA. 2,4-Dichlorophenoxyacetic acid and 4-amino-3,5,6-trichloropicolinic acid were completely ineffective. Besides culture media composition, the developmental stage of the explants at the time of culture showed a strong influence on somatic embryogenesis induction, with cotyledons from stage II pods providing the highest levels of induction. By contrast, the genotype of the explant did not determine a significant role in the induction process. Attempts to achieve somatic embryo germination were mostly unsuccessful, since only shoot development was observed; the highest frequencies of development occurred on media containing only gibberellic acid $(3.0 \mu M)$. For plant regeneration, the developed shoots were further rooted on IBA-supplemented media, and the plantlets obtained were transferred to soil, where $c$. $88 \%$ of them survived. Histological observations showed the presence of morphologically normal and abnormal somatic embryos, the latter displaying an abnormal pattern of vascular bundles. Ultrastructural analysis showed that the cells of the globular embryos had a dense cytoplasm, whereas those not involved in somatic embryo formation showed signs of senescence. Histological studies were also used to distinguish between somatic embryos and shoots originated in the same media.
\end{abstract}

Key words: Fabaceae; genotype; histology; rooting; ultrastructure.

\section{INTRODUCTION}

Ceratonia siliqua L. is a leguminous tree that grows spontaneously in some regions of Portugal (most notably the Algarve region) and in several other countries around the Mediterranean basin (Batlle and Tous, 1997). According to Batlle and Tous (1997), carob is one of the most interesting Mediterranean trees. From an ecological point of view, carob, like other leguminous trees, can be used for rehabilitation of marginal lands, due to its resistance to drought and salt tolerance (Lakshmi Sita, 1999). Currently, seed production is the main interest of carob growers. The endosperm of carob seeds produces a gum (carob beam gum) rich in galactomannans, which is used as a food additive (Batista, 1994). The pod has been traditionally used as a food stuff for humans and animals. A large number of carob cultivars have been reported around the world. In Portugal, the most frequent are Aida, Canela, Galhosa, and Mulata, which are appreciated for their high seed content (10-12\% of pod weight) and round kernels, well suited for gum extraction (Batlle and Tous, 1997). Portugal is one of

*Author to whom correspondence should be addressed: Email jorgecan@ bot.uc.pt the main producers of this gum (3600 ton $\left.\mathrm{yr}^{-1}\right)$, ranking fourth in the world after Spain, Italy, and Morocco.

Carob may be propagated through seeds or vegetatively. Seeds show good germination rates only after scarification. Furthermore, multiplication of seeds in dioecious species, does not ensure either the sex or the genetic characteristics of the cultivars. Propagation by cuttings is problematic because rooting is difficult (Lee et al., 1977). In this context, in vitro techniques offer an alternative to carob propagation (Romano et al., 2002). Micropropagation of carob through axillary meristem proliferation has been reported by several researchers (Sebastian and McComb, 1986; Romano et al., 2002). During the past two decades, somatic embryogenesis has been achieved in many leguminous trees, reviewed by Parrott et al. (1995), Lakshmi Sita (1999), and Trigiano et al. (1999). To our knowledge, the only report of somatic embryogenesis induction in carob is that of Carimi et al. (1997). These authors obtained low frequencies (4\%) of embryogenesis induction from fertilized ovules in Murashige and Skoog (MS) medium supplemented with $0.45 \mu M$ 2,4-dichlorophenoxyacetic acid (2,4-D), but no histological account of somatic embryo differentiation was given. According to Dunstan et al. (1995), histological information must be presented to confirm that the structures produced were in fact somatic embryos. This study reports 